\title{
Energy management system. Case study: hospital of hearth disease
}

\author{
Tirso L. Reyes Carvajal ${ }^{1}$, Juan Carlos Campos Avella ${ }^{2}$, João Evangelista Neto ${ }^{3}$, Ana Emília \\ Diniz Silva Guedes ${ }^{4}$
}

${ }^{1}$ Instituto de Tecnología y Educación de la Amazonia (ITEGAM), Manaos. Brasil.

${ }^{2}$ Universidad del Atlántico. Barranquilla. Colombia.

${ }^{3}$ Universidad del Estado del Amazonas (UEA). Manaos. Brazil.

${ }^{4}$ Universidade Federal de Rio Grande do Norte (UFRN). Natal. Brazil.

Email: tirsolrca@gmail.com, jcampos@e2energiaeficiente.com,joao_evangelista_neto@yahoo.com,aedsguedes@gmail.com

\author{
Received: October $23^{\text {th }}, 2016$ \\ Accepted: November $14^{\text {th }}, 2016$ \\ Published: December $22^{\text {th }}, 2016$ \\ Copyright @2016 by authors and Institute \\ of Technology Galileo of Amazon (ITEGAM). \\ This work is licensed under the Creative \\ Commons Attribution International \\ License (CC BY 4.0). \\ http://creativecommons.org/licenses/by/4.0/

\begin{abstract}
In this article is to describe the steps in the implementation of an energy management system and its application to a case study, this case was a hospital for cardiovascular diseases. The implementation of the energy management system in this center has been validated previously by the authors in many companies with different missions whether the productive sector and services. This method is result of several approach complementations from other existing methods, adding thermodynamics and mathematical-statistical tools. The aims of the method is to provide a tool that allows diagnose the energy management stage in enterprise or services center and at the same time address the enterprise energy management to achieving an efficient use of the energy carriers. The most important result of the implementation of the method in a hospital were the followings: Areas and equipment Higher Energy consumers, percentage of electricity not associated with patients serving directly, the ratio between the number of assist patients per month and the energy efficiency. These results allow know the potential savings in the hospital and establish an action plan.
\end{abstract}

Keywords: Energy management, thermodynamics and mathematical-statistical tools, Sequence for implementation of EMS.

\section{Sistema de Gestión Energética. Estudio de Caso: Hospital de Enfermedades Cardiovasculares}

\begin{abstract}
RESUMEN
En el presente artículo se describe los pasos a seguir en la implementación de un sistema de gestión energética y su aplicación a un caso de estudio, que en este caso fue un hospital de enfermedades cardiovasculares. La metodología de aplicación del sistema gestión energética en este centro ha sido validada con anterioridad por los autores en numerosas empresas del sector productivo como de los servicios. El principal objetivo del método de trabajo es proporcionar las herramientas matemáticoestadísticas y termodinámicas que permitan diagnosticar el nivel de la gestión energética en la organización como vía para lograr un uso eficiente de los portadores energéticos. Los resultados más importantes de la implementación del método en el estudio de caso fueron los siguientes: identificación y caracterización de áreas y equipos mayores consumidores de energía, determinación de la energía eléctrica consumida y no asociado con el servicio a los pacientes, la relación entre el número de camas día ocupadas y el consumo de energía.
\end{abstract}

Palabras claves: Gestión energética, herramientas matemático-estadísticas y termodinámicas, secuencia de aplicación del SGE 


\section{INTRODUCCIÓN}

La eficiencia energética forma parte de las políticas nacionales y es considerada la piedra angular para la obtención de altos niveles de racionalidad en el uso de la energía y un desarrollo sostenible.

Los defensores de eficiencia energética comúnmente citan una amplia gama de beneficios como son el uso racional de la energía, reducción de emisión de gases de efecto invernadero, disminuye la importación de portadores energéticos, incremento de la competitividad de las empresas y la creación de mayores fuentes de empleo [1]

Grandes han sido los esfuerzos durante las últimas cuatro décadas para medir la eficiencia energética y entender su impacto en el consumo de energía y diseñar sistemas eficaces de gestión energética. La Agencia Internacional de la Energía (AIE) ha jugado un papel de liderazgo en el desarrollo de indicadores energéticos para demostrar como el consumo de energía está ligado a las actividades humanas. Las políticas de eficiencia energética se han implementado en muchos de los países desarrollados [5], Sin embargo, los países más pobres y en vías de desarrollo aun carecen de recursos y herramientas que les permita avanzar con rapidez en este sentido.

Altos niveles de eficiencia energética basados en la identificación de pérdidas de energía, permiten una mayor disponibilidad de la energía para su uso. Se puede afirmar que la eficiencia energética es la fuente más barata de energía, ya que la inversión inicial ya fue hecha, son los equipos y tecnologías donde se producen las perdidas $[8,9]$. Es conocido que a nivel mundial se requieren 3 USD para reducir el consumo en $1 \mathrm{KWh}$ por eficiencia energética, mientras se invierten mínimo 6 USD para instalar una nueva capacidad de generar $1 \mathrm{KWh}$. (14) La aplicación de los sistemas de gestión energética nos permite determinar donde se encuentran estas pérdidas durante la operación de las instalaciones, su cuantificación, causas que las originan y las vías para su reducción o eliminación y el análisis costo-beneficio. Las formas de organizar los sistemas de gestión energética en las empresas o centros de servicio son variables dependiendo de las características de la organización, los intereses y la capacidad de los equipos de trabajo dentro de la empresa. Sin embargo, ya existe desde el año 2011 un estándar internacional que establece los requisitos mínimos que debe cumplir un sistema de gestión energética para que sea efectivo, basado en el modelo PHVA de la Organización Internacional de Estándares ISO denominado ISO 50.001. Sistemas de Gestión Energética.

Lo cierto es que todos están de acuerdo que el incremento de la eficiencia energética es la clave del éxito de la organización.

La implementación del sistema de gestión energética requiere de un sistema de información por área de producción o centro de costo, lo que implica la medición de los consumos. El registro del consumo histórico de energía es una información clave, para cualquier tipo de organización,

Uno del problema que se presentan en la realidad de la producción o los servicios para la implementación de un sistema de gestión energética es que las instalaciones o áreas no cuentas con dispositivos propios de medición de consumos ni evaluación de la calidad de la energía consumida [8]. Muchas plantas o centros de servicio sólo tienen un único metro medidor que contabiliza el consumo total y no por zonas. Por eso a veces sólo es posible determinar el consumo total de la organización, pero no para las áreas de producción o máquinas que representan el mayor consumidor de energía y generalmente es donde se encuentran los mayores potenciales de ahorro. Es por ello que antes de comenzar con la implementación de un sistema de gestión energética, el sistema de información de los consumos de energía de la organización debe ser revisado para establecer la estrategia del trabajo a realizar con el fin de garantizar la disponibilidad de toda la información necesaria.

\section{MATERIALES Y MÉTODOS}

El objetivo del método es poner en práctica medidas eficaces en el lugar que proporcionan ahorros significativos de energía. En la práctica un sistema de gestión energética debería dar como resultado, los siguientes:

Monitoreo del desempeño energético de las instalaciones y control de los consumos.

Programa de motivación y formación especializada de los recursos humanos que mayor impacto puede tener en el uso eficiente de la energía.

Propuestas de proyectos para la mejora de la eficiencia energética en el corto, mediano y largo plazos.

La Figura 1 muestra las actividades relacionadas con las etapas técnicas y procesos del sistema son $[6,9]$ :

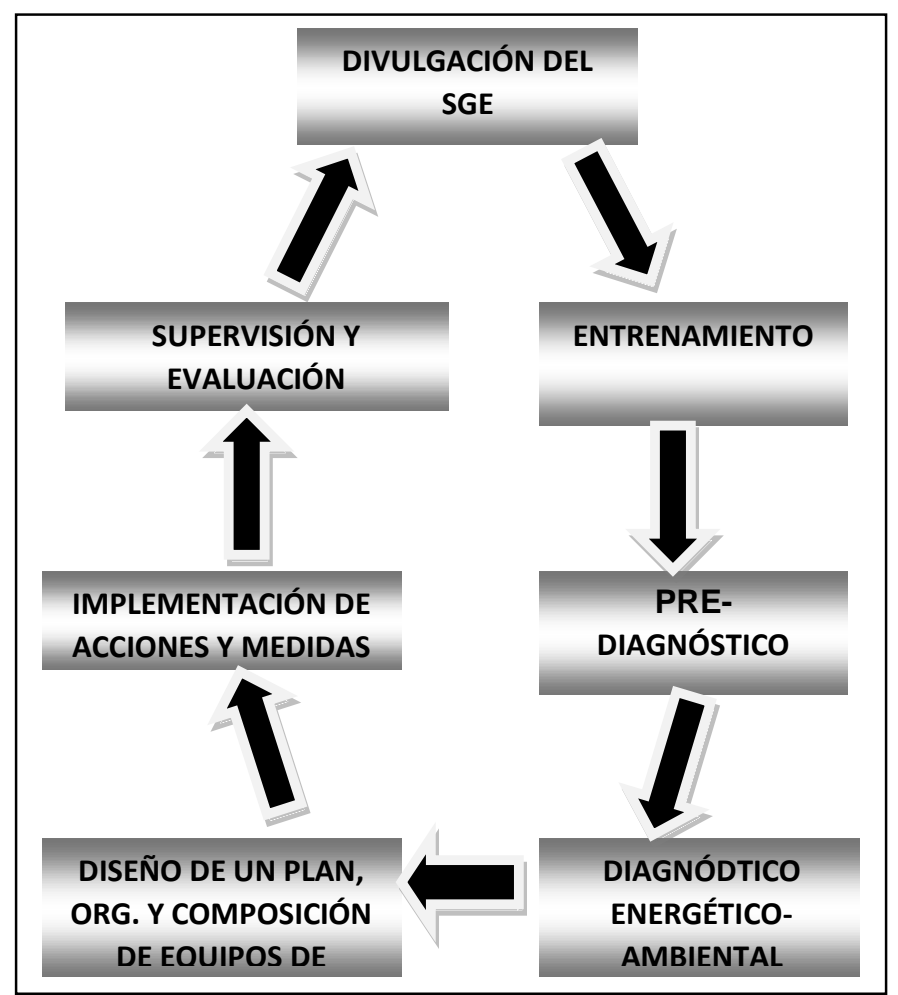

Figura 1. Secuencia de aplicación del sistema de gestión energética

Fuente: Los Autores, (2016).

Las iniciativas o actividades que tienen lugar en la empresa deben ser consultadas y aprobadas por la alta gerencia de 
la empresa, conocer el desarrollo de la iniciativa y los resultados a obtener. El objetivo central de la gerencia es mantener a la empresa dentro de parámetros aceptables y rentables en su gestión lográndose un equilibrio entre la iniciativa y el control. Formulación de posibles objetivos y propuesta de alternativas dentro de los posibles escenarios de acuerdo a la misión de la organización.

Por esta razón, la alta gerencia de la empresa juega un papel relevante cuando se inicia la implementación del sistema de gestión energética.

\section{II.1 DIVULGACIÓN DEL SISTEMA DE GESTIÓN ENERGÉTICA}

Promover y difundir la importancia de implementar el sistema de gestión energética en la empresa con el fin de hacer de la energía un uso más racional como premisa para un mejor desempeño de la empresa. El primer paso es obtener la aprobación de la alta dirección de la empresa.

\section{II.2 ENTRENAMIENTO}

La capacitación de los recursos humanos, la sensibilización y las campañas publicitarias regulares.

Este personal tendrá una participación directa en la implementación del sistema de gestión energética en la empresa.

\section{II.3 PRE-DIAGNÓSTICO}

$\checkmark$ Balance del consumo y uso final de la energía

$\checkmark \quad$ Identificación y análisis de índices globales.

$\checkmark$ Gráficos de comportamiento del consumo.

$\checkmark$ Diagnóstico preliminar energético-ambiental.

$\checkmark$ Diagnóstico general al sistema de gestión energética.

$\checkmark \quad$ Identificación de los potenciales de ahorro energético.

\section{II.4 DIAGNÓSTICO ENERGÉTICO-AMBIENTAL}

$\checkmark \quad$ Estructura de consumo y uso final de la energía

$\checkmark$ Estructura de consumo y costos de portadores energéticos primarios y secundarios.

$\checkmark \quad$ Identificación de las áreas, equipos y personal que mayor influencia tiene sobre los consumos de energía.

$\checkmark$ Identificación de los parámetros y actividades de operación y de mantenimiento que mas impactan los consumos de energía

$\checkmark$ Costo de los flujos primarios y secundarios de energía.

$\checkmark$ Estructura de las pérdidas de energía.

$\checkmark \quad$ Identificación de las prácticas ineficientes.

$\checkmark \quad$ Mecanismos de evaluación de interés.

$\checkmark$ Determinación de los niveles de competencia.

$\checkmark \quad$ Identificar las oportunidades y los ahorros potenciales.

$\checkmark$ Relación de problemas.

\section{II.5 DISEÑO DE UN PLAN, ORGANIZACIÓN. Y COMPOSICIÓN DE EQUIPOS DE MEJORA \\ $\checkmark$ Identificación de soluciones. \\ $\checkmark$ Evaluación Técnico- Económica.}
$\checkmark$ Establecimiento de escenarios.
$\checkmark$ Clasificación de soluciones.
- Planificación de soluciones y metas.
- Diseño del Sistema de Monitoreo.
- Diseño y conocimiento del programa de entrenamiento.
$\checkmark$ Definir estructuras necesarias.
$\checkmark \quad$ Identificar los roles y misiones.
$\checkmark$ Sistemas de retroalimentación.
$\checkmark$ Mecanismos de estimulación.
$\checkmark$ Barreras y alternativas.

\section{II.6 IMPLEMENTACIÓN DE ACCIONES Y MEDIDAS}

Obtener el apoyo y la cooperación de las personas clave en diferentes niveles dentro de la organización es un factor importante para la exitosa implementación del plan de acción.

Alcanzar las metas del sistema depende con frecuencia de la conciencia, el compromiso, el conocimiento y la capacidad de las personas que van a poner en práctica los proyectos. La ejecución del plan de acción llevará tiempo.

Para poner en práctica un plan de acción, deben adoptarse las siguientes medidas:

- Desarrollar la información específica para las audiencias claves sobre el programa de gestión energética.

- Fortalecer el apoyo en todos los niveles de la organización a las iniciativas y objetivos de la gestión energética

- A través de la formación, el acceso a la información, procedimientos y tecnologías así como la transferencia de prácticas exitosas, se puede ampliar el conocimiento y la capacidad de las personas involucradas.

\section{II.7 SUPERVISIÓN Y EVALUACIÓN}

$\checkmark$ Establecimiento de criterios de control operativo y de mantenimiento de los parámetros y actividades que impactan el consumo.

$\checkmark$ Seguimiento y Evaluación del desempeño energético a través de los índices.

$\checkmark$ Identificación de las de las desviaciones del índice respecto a su valor deseado

$\checkmark \quad$ Identificación y análisis de las causas de las desviaciones.

$\checkmark$ Implementación del sistema de correcciones.

$\checkmark \quad$ La divulgación de resultados.

La aplicación del sistema de gestión energética en la empresa se puede estimar entre 6 meses a 1 año. El sistema de gestión energética se basa en herramientas matemático-estadística y termodinámicas para su aplicación, con la filosofía de Gestión Total de la Calidad $[2,6,8,10]$.

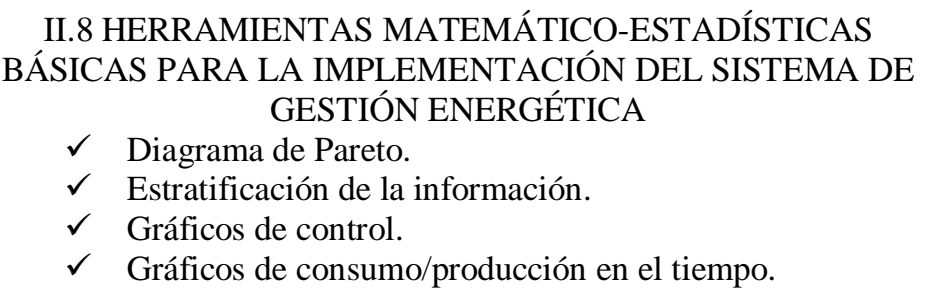


$\checkmark$ Gráfico Consumo-Producción (diagrama de correlación)

$\checkmark$ Gráfico de tendencia de las desviaciones del consumo energético en el tiempo

$\checkmark$ Gráficos de Índice de consumo-Producción

$\checkmark$ Gráficos de comportamiento del indicador del desempeño energético en el tiempo.

$\checkmark$ Gráficos de seguimiento de las desviaciones del presupuesto de energía.

$\checkmark$ Gráficos de correlación de la tendencia del desempeño energético con la variación de los parámetros y actividades de operación y mantenimiento que impactan el consumo energético.

\section{II.9 HERRAMIENTAS TERMODINÁMICAS MÁS UTILIZADAS EN LOS DIAGNÓSTICOS ENERGÉTICO- AMBIENTALES:}

$\checkmark$ Diagrama de flujos energéticos.

$\checkmark$ Balance de masa y energía

$\checkmark$ Análisis entrópico y exergético.

$\checkmark$ Análisis termoeconómico

$\checkmark$ Otras herramientas

\section{ANÁLISIS DE RESULTADOS}

La presente metodología ha sido aplicada en diferentes organizaciones del sector industrial y de los servicios con resultados satisfactorios lo que ha quedado demostrado a través de los posibles potenciales de ahorro disponibles en diferentes instalaciones.

A continuación, enumeraremos algunos de los resultados obtenidos en un estudio de caso: Hospital de Enfermedades Cardiovasculares

El Diagrama de Pareto, Figura 2, es la herramienta recomendada para el establecimiento de la estructura de consumo, un gráfico en forma de barras que clasifica en forma descendente los factores que se analizan (Consumo de portadores energéticos) en función de su frecuencia, importancia absoluta o relativa. Adicionalmente permite observar en forma acumulada la incidencia total del parámetro objeto de análisis.

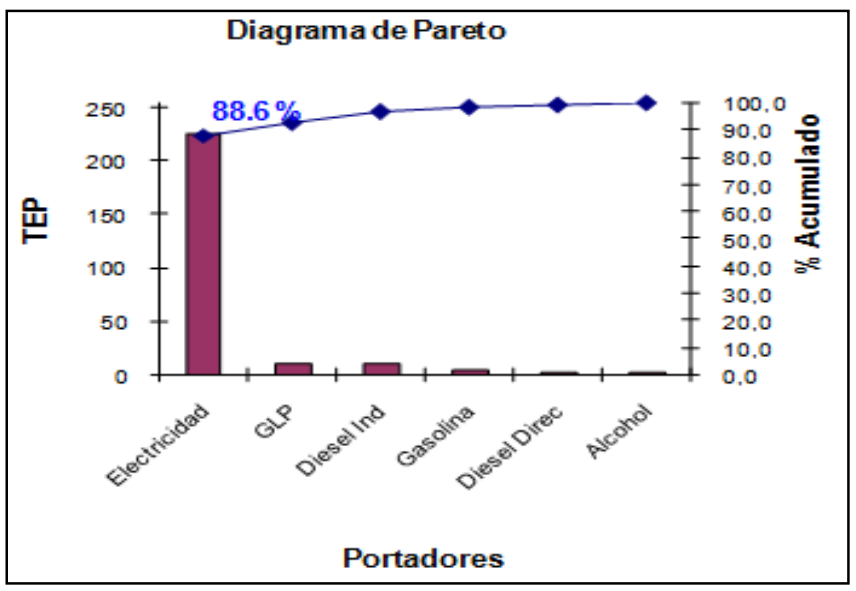

Figura 2. Diagrama del Pareto

Fuente: Los Autores, (2016).
De acuerdo al análisis de Pareto se obtuvo que el consumo de electricidad represente el $86,6 \%$ del total de los portadores energéticos usados en el hospital, por lo que los mayores potenciales de ahorro se concentran en el portador electricidad.

El desglose del portador energético electricidad por área de trabajo ayuda a entender donde y como es usada la energía eléctrica en el hospital, además de poder descubrir donde pueden estar los mayores potenciales de ahorro, Figura 3

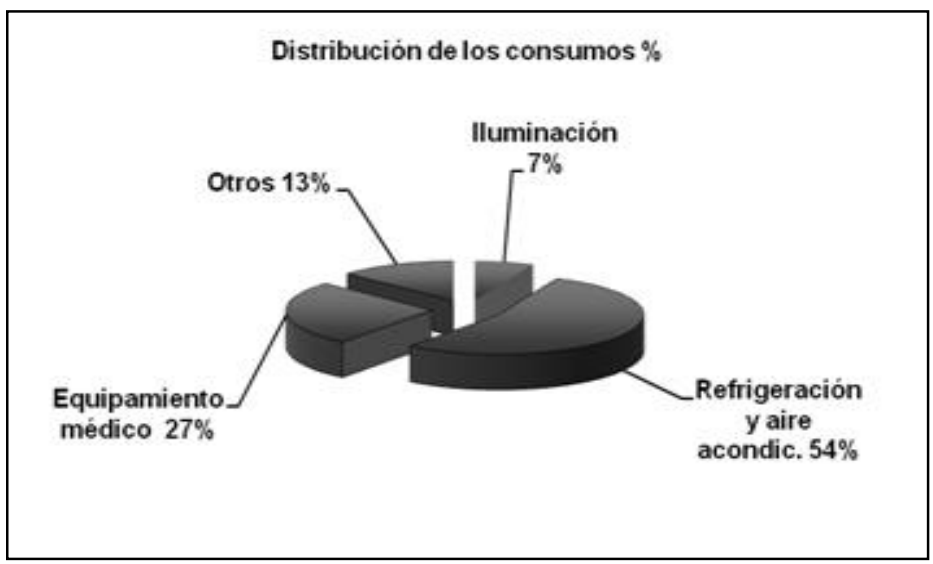

Figura 3. Distribución del consumo de la electricidad \%.

Fuente: Los Autores, (2016).

\section{III.1 ESTRATIFICACIÓN DE LA INFORMACIÓN}

La estratificación es el método de agrupación de los datos asociados por puntos o características comunes pasando de lo general a lo particular. (La aplicación de la ley de Pareto, sucesivamente), y proporciona información sobre el equipo y las áreas donde se consume la mayor cantidad de energía.

La estratificación de la información permite:

- Discriminar las causas que están provocando el efecto estudiado.

- Conocer el árbol de causas de un problema o efecto.

- Determinar la influencia cuantitativa de las causas particulares sobre las generales y sobre el efecto estudiado.

Como resultado de la estratificación se obtuvo:

El sistema centralizado de aire acondicionado y la sala de hemodinámica representan el $23 \%$ y el $18 \%$ respectivamente de todo el consumo energético.

\section{III.2 GRÁFICO CONSUMO-SERVICIO (DIAGRAMA DE CORRELACIÓN)}

Este diagrama (E vs $\mathrm{S}$ ) debe ser realizado por tipo de portador energético, y por área, teniendo en cuenta en cada caso la producción o servicio asociado con el portador energético en cuestión, Figura 4. 


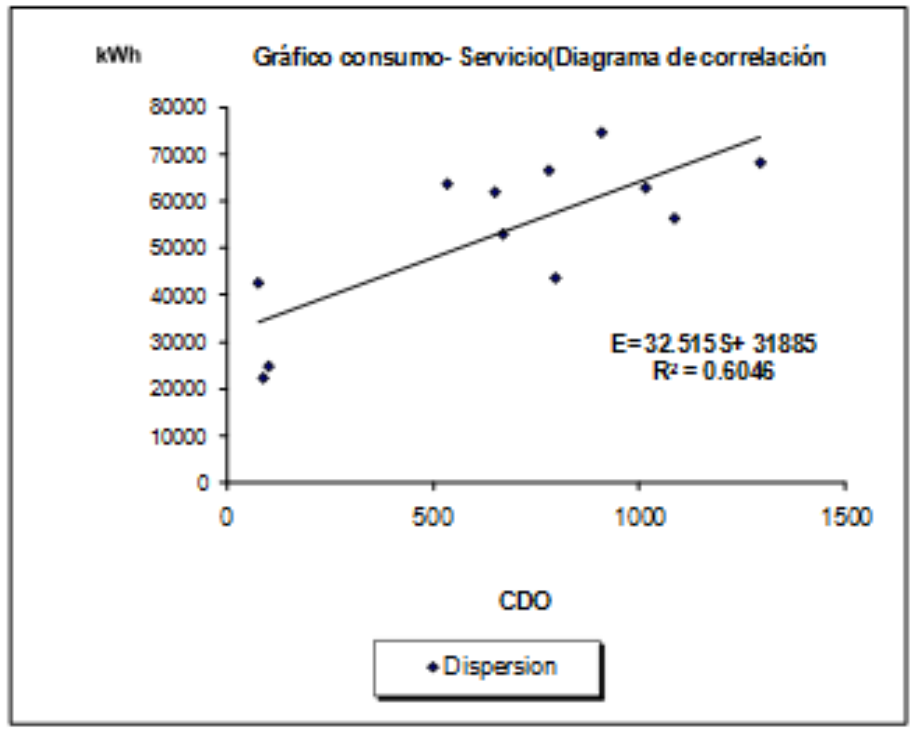

Figura 4. Gráfico de la correlación energía vs cama día ocupada (E vs CDO).

Fuente: Los Autores, (2016).

Según la figura anterior existen dos pares de puntos, uno al comienzo y otro en la parte central del gráfico con similar cantidad de camas día ocupadas y diferente valor de energía consumida correspondientes a los pares de meses enero-marzo y septiembre-diciembre lo cual viene dado por el hecho de que la carga instalada en equipos de climatización y refrigeración representa el 53\% del total y la correlación entre el tiempo de trabajo de este tipo de carga y la temperatura es elevado, de ahí que en los meses de marzo y septiembre se haya consumido más energía eléctrica con similar cantidad de camas día ocupadas que en los meses de enero y diciembre respectivamente.

La energía consumida (kW-h) está relacionada con las camas día ocupadas por la expresión:

$$
\mathrm{E}=\mathrm{m} \cdot \mathrm{CDO}+\mathrm{E}=32,5 \mathrm{CDO}+\mathrm{Eo}
$$

Donde:

E- Energia consumida $(\mathrm{kW}-\mathrm{h})$

CDO - Cama día ocupada

Eo - Energía no asociada al servicio (kWh)

M - Pendiente de la recta

m.CDO -Energía asociada al servicio $(k W h)$

El nivel de confianza o grado de probabilidad $\left(\mathbf{R}^{2}\right)$ del modelo matemático anterior es del $60,5 \%$, resultando bajo, dado por múltiples factores, entre otro, la falta de control en el uso de la energía, como uno de los más importantes.

El porciento de energía no asociado a los servicios es de un $\mathbf{5 9 , 5 \%}$ relacionada fundamentalmente a las áreas de oficinas médicas, no médicas y administrativas que tienen una carga significativa de aires acondicionados, equipos de cómputo e iluminación trabajando durante las ocho horas laborables del día.

Después de haber obtenido el gráfico E vs CDO y la ecuación que los relaciona, pasaremos a realizar el diagrama de índice de consumo vs CDO, Grafico 5, de gran utilidad para establecer sistemas de gestión energética y estandarizar procesos a niveles de eficiencia energética superiores ya que permite determinar el punto crítico de producción o de servicios de una entidad.

\section{III.3 GRÁFICO ÍNDICE DE CONSUMO - PRODUCCIÓN.}

La función $\mathrm{IC}=\mathrm{f}(\mathrm{S})$ se obtiene como sigue:

$\mathrm{E}=\mathrm{m} \cdot \mathrm{S}+\mathrm{Eo}$

$\mathrm{E} / \mathrm{S}=\mathrm{m} . \mathrm{S} / \mathrm{S}+\mathrm{Eo} / \mathrm{S}$

$\mathrm{IC}=\mathrm{m}+\mathrm{Eo} / \mathrm{S}$

E: Energía

S: Servicio (Producción)

m: Pendiente. La pendiente de la línea de 32,5 Representa el consumo de energía adicional por el servicio

Eo: La energía no asociada con el servicio (perdida de energía).

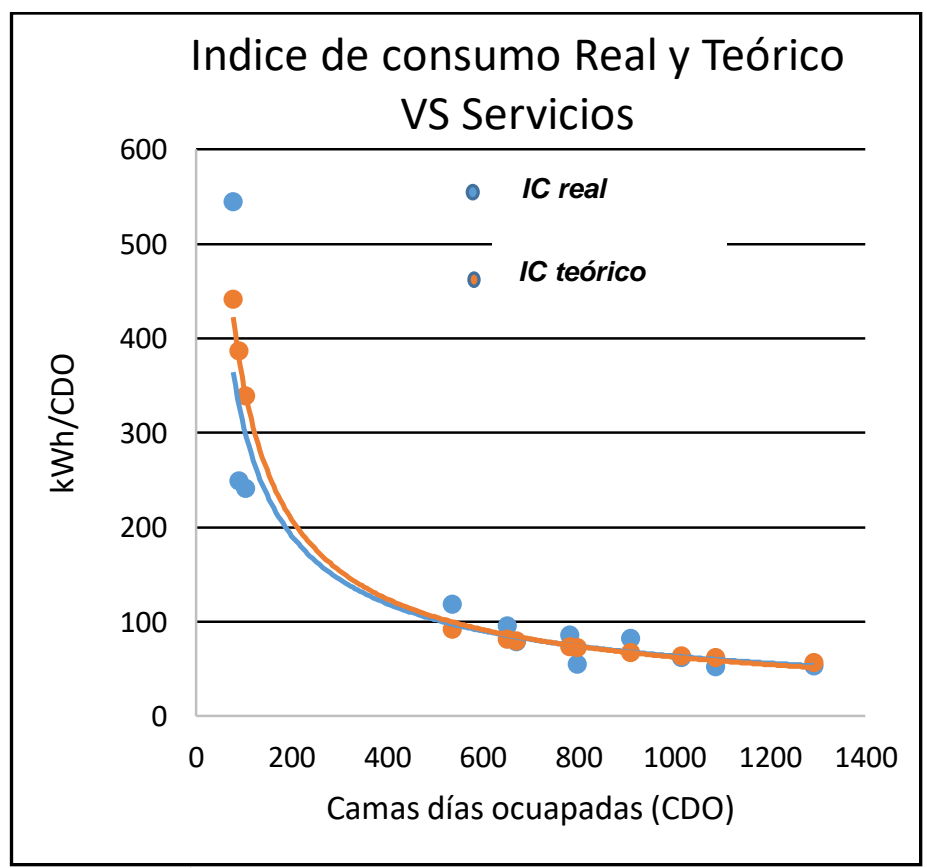

Figura 5. Índice del consumo.

Fuente: Los Autores, (2016).

Valores de IC por debajo de la curva que representa el comportamiento del índice durante el período de referencia comparativa, indican un incremento de eficiencia del proceso, en el caso contrario existe un potencial de disminución del índice de consumo igual a la diferencia entre el IC real y el IC teórico para igual nivel de servicio, Tabla 1. 
Tabla 1: Comparación entre IC real y IC teórico para igual CDO.

\begin{tabular}{|c|c|c|c|c|c|}
\hline \multicolumn{7}{|c|}{$\begin{array}{c}\text { Ecuación de correlación para el caso estudiado: } \\
\text { E = 32,515*S + 31885 }\end{array}$} \\
\hline Meses & $\begin{array}{c}\text { Consumo } \\
\text { real }\end{array}$ & CDO & $\begin{array}{c}\text { IC } \\
\text { real }\end{array}$ & $\begin{array}{c}\text { Consumo } \\
\text { teórico }\end{array}$ & IC teórico \\
\hline Enero & 22410 & 90 & 249 & 34811,4 & 386,8 \\
\hline Febrero & 25064 & 104 & 241 & 35266,6 & 339,1 \\
\hline Marzo & 42510 & 78 & 545 & 34421,2 & 441,3 \\
\hline Abril & 52930 & 670 & 79 & 53670,1 & 80,1 \\
\hline Mayo & 74456 & 908 & 82 & 61408,6 & 67,6 \\
\hline Junio & 68476 & 1292 & 53 & 73894,4 & 57,2 \\
\hline Julio & 63665 & 535 & 119 & 49280,5 & 92,1 \\
\hline Agosto & 62496 & 651 & 96 & 53052,3 & 81,5 \\
\hline Sept. & 67166 & 781 & 86 & 57279,2 & 73,3 \\
\hline Octubre & 62930 & 1015 & 62 & 64887,7 & 63,9 \\
\hline Nov. & 56420 & 1085 & 52 & 67163,8 & 61,9 \\
\hline Dic. & 43835 & 797 & 55 & 57799,5 & 72,5 \\
\hline
\end{tabular}

Fuente: Los Autores, (2016).

De los resultados anteriores podemos apreciar que cinco meses tuvieron un comportamiento por encima de la curva teórica, contándose con un ahorro de haberse disminuido los índices de consumo reales para estos meses hasta el valor teórico de aproximadamente $55000 \mathbf{~ k W h / a n ̃ o . ~}$

En el gráfico de $I C$ vs $C D O$ existe un punto (punto crítico) donde comienza a elevarse significativamente el IC para bajas cifras de $\mathrm{CDO}$ con los siguientes valores: $\mathbf{C D O}=\mathbf{7 8 5}$ (camas día ocupada); IC = 70 kWh $/$ CDO

Por encima 785 camas día ocupadas para un mes no cambia significativamente el índice de consumo, sin embargo, por debajo de este valor se incrementa rápidamente disminuyendo la eficiencia energética del centro.

Las oscilaciones del consumo en el tiempo referido a los serviços puede ser observado el Gráfico de la figura 6.

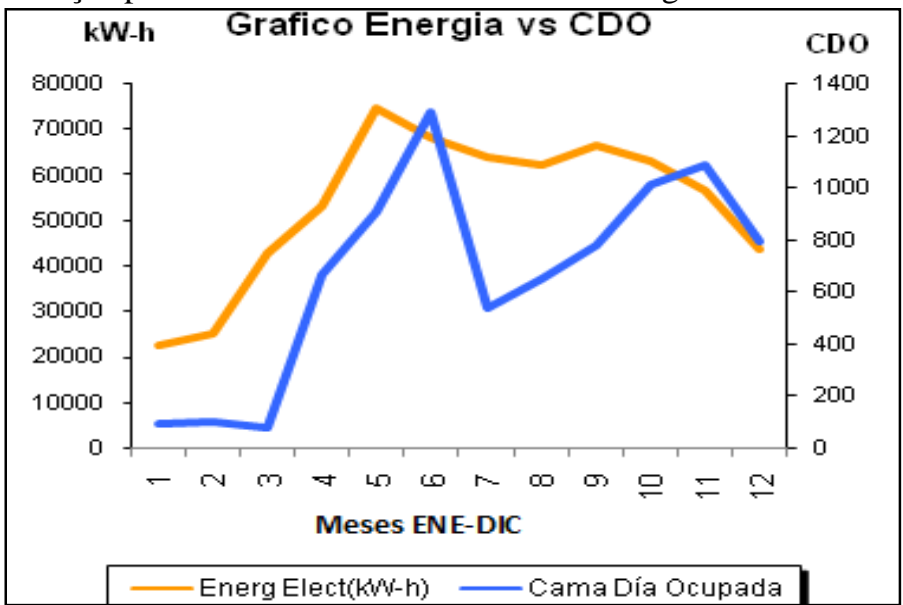

Figura 6. Gráfico del consumo de energía vs cama día ocupada en el tiempo (E vs CDO)

Fuente: Los Autores, (2016).
Realizando un análisis general del gráfico anterior podemos observar que no existe un comportamiento proporcional en todo el período, con algunas incongruencias en determinados meses donde hay incrementos de consumo de energía y decrecimiento de las camas día ocupadas.

Para clasificar los puntos del gráfico con un comportamiento anómalo se tuvieron en cuenta las siguientes condiciones:

1. Incremento de la energía y decrecimiento de CDO.

2. Decrece el consumo de energía y se incrementan las CDO.

3. La razón de variación de la energía y las CDO, ambos creciendo o decreciendo, son significativos en el período analizado.

De forma general podemos evaluar el comportamiento del consumo de energía y las CDO como anómalo pues solo el $45 \%$ de los puntos graficados sigue un comportamiento normal.

\section{4 GRÁFICO DE CONTROL DE ENERGÍA ELÉCTRICA}

La mayor parte de los procesos de servicios tienen un comportamiento denominado normal, es decir, existe un valor medio del parámetro de salida muy probable de obtener y a medida que nos alejamos de este valor medio la probabilidad de aparición de otros valores de este parámetro cae bruscamente si no aparecen causas externas, Grafico de la figura 7.

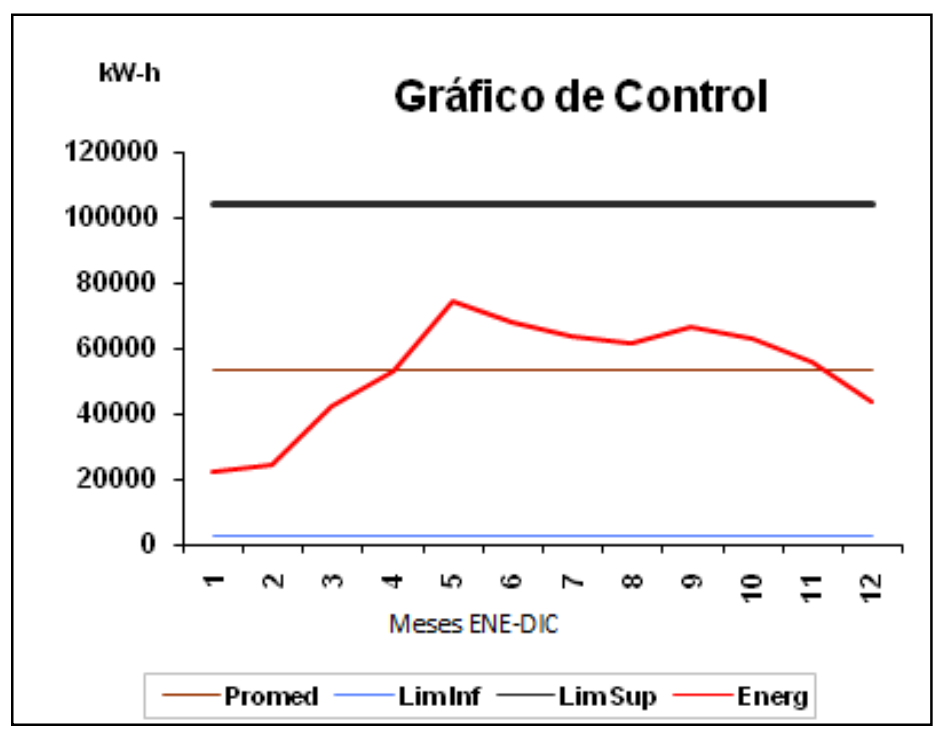

Figura 7. Gráfico del Diagrama de control.

Fuente: Los Autores, (2016).

Del gráfico anterior se puede observar como los consumos de energía eléctrica en los dos primeros meses del año estuvieron distanciados del valor medio. El motivo de dicho comportamiento está en el hecho de que no se realizaron operaciones en este período por labores de reparaciones en el centro, solo se realizaron estudios de hemodinámica y de electrofisiología. 


\section{CONCLUSIONES}

$\checkmark$ La implementación de las herramientas del sistema de gestión energética por primera vez en el caso de estudio es clave para conocer el escenario energético actual y la forma de diseñar un plan que posibilite una mayor eficiencia energética, así como para la sensibilización de directivos y empleados en el uso racional de la energía. Los resultados obtenidos son una referencia para el seguimiento y control del funcionamiento de la organización, así como para el establecimiento de procedimientos y reglamentos para la aplicación de las medidas adoptadas.

$\checkmark$ Las herramientas implementadas durante el caso de estudio lograron el soporte requerido para el compromiso de la alta dirección en el monitoreo y control del consumo energético requerido para el funcionamiento de la organización

$\checkmark \quad$ Para el estudio de caso la energía eléctrica representa el $89,3 \%$ del total de los consumos de portadores energéticos.

$\checkmark$ La climatización, refrigeración y equipos médicos representan el $80 \%$ de los consumos de energía eléctrica.

$\checkmark$ El punto operativo de mayor eficiencia para la sala de hemodinámica se corresponde con 225 intervenciones/mes, $40 \mathrm{kWh} / \mathrm{int}$. y para las salas de operaciones 56 int. quirúrgicas/mes con un gasto energético de $155 \mathrm{kWh} / \mathrm{int}$.

$\checkmark$ La energía no asociada directamente al servicio a pacientes es de $59.5 \%$

$\checkmark$ El punto operativo de mayor eficiencia del hospital es $785 \mathrm{CDO} / \mathrm{mes}$ con un índice de consumo de 70 $\mathrm{kWh} / \mathrm{CDO}$.

$\checkmark$ Para el caso del gas del GLP existe un consumo no asociado a la elaboración de alimentos que representa el $67 \%$ del total del gas.

$\checkmark$ Por debajo de las 8350 raciones elaboradas por mes el consumo de gas se eleva considerablemente por unidad de ración elaborada.

$\checkmark \quad$ El $43 \%$ del agua que se consume se pierde en salideros, anualmente el hospital pudiera ahorrar un estimado de 4500 USD por este concepto.

\section{BIBLIOGRAFÍA}

[1] Schnapp, R. Energy statistics for energy efficiency indicators. Joint Rosstat-IEA Energy Statistics Workshop Moscow, February 2012.

[2] Barney L. Capehart, Wayne C. Turner, Guide to Energy Management, Seventh Edition Hardcover- August 18, 2011.

[3] By Katrina Buff, Association of Energy Engineers. 2003 Energy \& High Performance Facility Sourcebook. Published by CRC Press.
[4] O. Ray Whittington, Patrick R. Delaney. 2007. Wiley CPA Exam Review: Business Environment and Concepts. Published by John Wiley and Sons.

[5] International Energy Agency. Implementing energy efficiency policies. Are IEA member countries on track. 2008.

[6] Grupo de Investigación en GEE, KAÍ, Universidad del Atlántico. Grupo de Investigación en Energías, GIEN, UAO. Sistema de Gestión Integral de la Energía. Guía para la implementación. ISBN 978-958-8123-43-1. Noviembre, 2008. Colombia.

[7] G. J. Levermore. 2000. Building Energy Management Systems: Applications to Low-energy HVAC and Natural Ventilation Control. Published by Taylor \& Francis.

[8] Reyes Carvajal T.L. Colectivo de autores. 2007. Caracterización energética del sector empresarial en la provincial Villa Clara. Dpto. Energía. Universidad Central de las Villas. Cuba.

[9] Borroto Nordelo A. Colectivo de autores. 2002. Gestión Energética Empresarial. CEEMA. Universidad de Cienfuegos. Cuba.

[10] Campos Avella J. C. 2008. Caracterización energética. http://ecodesarrollo.cl.

[11] Pieter-Jan Stockmans. April 2007. Energy management: self-assessment. Laborelec. http://www.leonardo-energy.org.

[12] Sustainable Energy Ireland. December 2006. Energy Management Systems Technical Guideline. http://www.sei.ie/getFile.asp?FC_ID=2263\&docID=838

[13] Intelligent Energy - Europe (EIE). June 2005. EIE/04/246/S07.38678. Benchmarking and Energy Management Schemes in http://alpha.cres.gr/bess/downloads/BESS_WP_1_final_report_s ummary.pdf.

[14] Capturing the Multiple Benefits of Energy Efficiency, 232 pages, ISBN 978-92-64-22072-0 